\title{
Incidence of Wound Dehiscence Following Obstetric and Gynecological Surgeries at a Tertiary Care Hospital: A Retrospective Study
}

\author{
Mrityunjay C Metgud ${ }^{1}$, Anubha Kataria², Spoorthi R Nadipally ${ }^{3}$, Kamal Patil ${ }^{4}$
}

\begin{abstract}
Aim: A retrospective analysis with an aim to find out the incidence of wound dehiscence following obstetric and gynecological surgeries was carried out at a referral hospital and to review some of the practices in the hospital surgical and postoperative care of the surgical wound. Materials and methods: This study was carried out in the Department of Obstetrics and Gynecology at KLEs Dr Prabhakar Kore Charitable Hospital, a tertiary referral hospital in Belagavi, Karnataka, India, from May 2016 to August 2017 by obtaining data from medical records in the hospital. Results: During the study period, 3,172 women underwent major obstetric and gynecological surgeries; out of which 97 women developed wound dehiscence (3.05\%). A total of 82 women had wound dehiscence following obstetric surgeries (84.54\%) and 15 women following gynecological surgeries (15.46\%). The number of emergency surgeries were 79 (81.44\%), which included emergency lower segment cesarean section (LSCS) and the elective cases were 18 (18.56\%), which comprised of elective LSCS, tubal ligation, and gynecological surgeries.

Conclusion: The aim of this study was to review some of the practices during surgery and postoperative care in our hospital. The practice of scrubbing the abdomen prior to the day of surgery was followed. Subcutaneous fat suturing before closing the skin with subcuticular sutures was never practiced even when the fat layer was quite thick. Dressing was routinely changed on the 3rd day in the postnatal ward (exposing the wound to dirty linen particles when the wound had still not healed). However, preoperative antibiotics were routinely given prior to incision and also postoperative antibiotics for a total of 5 days.

Clinical significance: Intraoperative and postoperative factors are significant in wound healing in addition to patient factors. Dressing should probably be done first only on the 5th postoperative day, which may prevent introduction of infection.

Keywords: Dehiscence, Retrospective study, Wound dehiscence.

Journal of South Asian Federation of Obstetrics and Gynaecology (2020): 10.5005/jp-journals-10006-1763
\end{abstract}

\section{INTRODUCTION}

A wound is an interruption in the permanence of the soft parts of the body structures. ' Both endogenous or exogenous flora can infect a surgical wound. ${ }^{2}$ In spite of a range of advances made in the field of asepsis, antimicrobial drugs, operative methods, and sterilization, the postoperative wound problems continue to be a foremost threat. ${ }^{3}$ Appraisal of factors responsible for surgical site infection in obstetric patients is important. ${ }^{4-6}$ Strategiesfor prevention of wound infections have been suggested from time to time. ${ }^{7}$ The rising incidence of wound gape in the postoperative period is a humungous challenge to the obstetricians and gynecologists despite many amendments in the protocols for the preoperative, intraoperative, and postoperative management of the patient time and again. Consequences of post-cesarean section wound infection can be the discharge from suture line, wound gaping, and can be upsetting resulting in burst abdomen. ${ }^{8}$ Considering that the overall basic standards are ideally maintained in all tertiary care hospitals, a retrospective study was done to measure the incidence of wound gape in the postoperative period and the contributing risk factors along with the common causative organisms. This particular study is a prelude to the prospective study to be done to know the importance of measures like suturing subcutaneous fat before approximating the skin, keeping drain in situ in obese women, and avoiding changing of dressing in the wards prior to the 5th postoperative day.

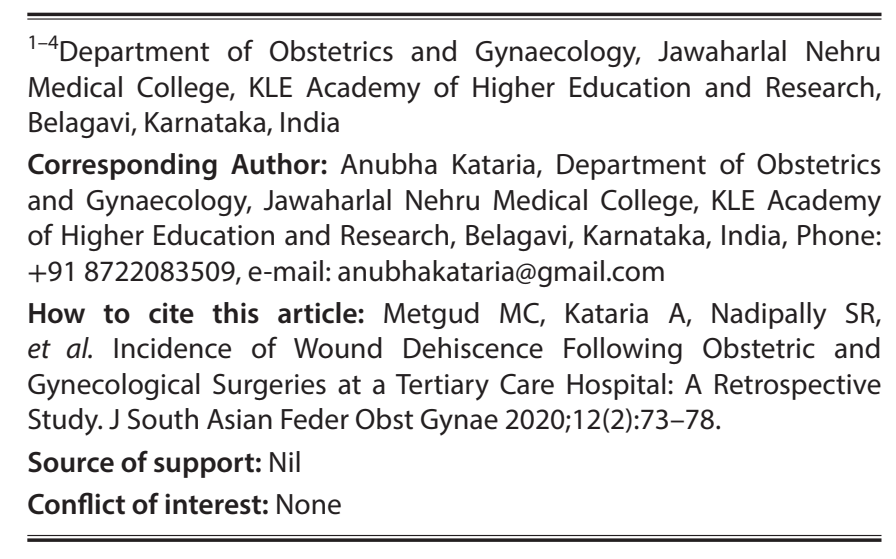

\section{Materials and Methods}

\section{Setting}

This study was a retrospective observational study carried out in the department of obstetrics and gynecology at a tertiary care hospital attached to KLEs Dr Prabhakar Kore Charitable Hospital in Belagavi, Karnataka, India. This is a 1,250-bed tertiary care hospital attached to KAHER's Jawaharlal Nehru Medical College and one of the major referral centers in the northern part of Karnataka in India.

\section{Duration}

The study was conducted during a period of May 2016-August 2017.

(c) The Author(s). 2020 Open Access This article is distributed under the terms of the Creative Commons Attribution 4.0 International License (https://creativecommons. org/licenses/by-nc/4.0/), which permits unrestricted use, distribution, and non-commercial reproduction in any medium, provided you give appropriate credit to the original author(s) and the source, provide a link to the Creative Commons license, and indicate if changes were made. The Creative Commons Public Domain Dedication waiver (http://creativecommons.org/publicdomain/zero/1.0/) applies to the data made available in this article, unless otherwise stated. 


\section{Inclusion Criteria and Exclusion Criteria}

All the patients who underwent major obstetric and gynecological surgeries in KLEs Dr Prabhakar Kore Charitable Hospital in Belagavi, Karnataka, India, from May 2016 to August 2017 were included in the study. The procedures included were both emergency and elective cases. Emergency cases included emergency lower segment cesarean section (LSCS) carried out in the labor room operation theater, and elective cases included elective LSCS, abdominal tubectomy, and major gynecological surgeries (abdominal hysterectomy, exploratory laparotomy, or tumor excision) done in the routine operation theater for elective cases. Laparoscopic surgeries, fractional curettage, and medical termination of pregnancy were excluded.

All the patients undergoing surgery were given preoperative antibiotics in the form of intravenous ceftriaxone $1 \mathrm{~g} \mathrm{IV}$. The surgical site was painted adequately with betadine solution (10\%) followed by alcohol. All surgical closures in primary surgery were done by vicryl (polyglactin). The rectus sheath was closed with polyglactin no.1. Skin was approximated by subcuticular sutures using polyglactin 3-0/polyglactin 2-0. However, in none of the patients subcutaneous fat was approximated. The first wound dressing postoperatively was done on the 2 nd or 3 rd day in the wards.

\section{Data Collection}

Data were collected retrospectively from the medical records in the operation theater and wards. This data included the patient demographics, date of admission, indication for surgery, date and type of surgery, already existing comorbidities in the patient, type of sutures used to close the wound site, an duration of stay in the hospital for primary surgery and then for secondary suturing. Also data were collected from the microbiology laboratory regarding organisms grown on culture from the swab taken from the wound dehiscence site. Ethical clearance for the study was obtained from the institutional ethics committee.

\section{Statistical Analysis}

The statistical analysis in this study was simple tabulation of each variable along with its frequency in wound dehiscence infections. Then a comparative univariate analysis of these variables was done, which led to a simple calculation of percentage. All women who underwent the abovementioned surgeries in these particular operation theaters were followed up and the number of women who developed wound dehiscence was used to calculate the incidence of wound gape (primary outcome), expressed in terms of percentage. All the secondary outcomes were calculated using univariate analysis and results were expressed in the form of percentage.

\section{Results}

\section{Incidence of Wound Dehiscence}

A total of 3,172 women underwent major obstetric and gynecological surgeries. Out of these 97 women developed wound dehiscence, with an incidence of wound dehiscence as 3.05\%. All 97 women underwent secondary suturing. Among these 97 women, 82 women had wound dehiscence following obstetric surgeries (84.54\%) and 15 women following gynecological surgeries (15.46\%) (Table 1). The number of emergency surgeries was $79(81.44 \%)$, which included emergency LSCS, and the elective cases were 18 (18.56\%), which comprised of elective LSCS, tubal ligation, and gynecological surgeries (Table 2).
Table 1: Percentage of wound dehiscence in different types of surgeries

\begin{tabular}{ll}
\hline Surgery & Percentage $(n=97)$ \\
\hline Gynecological & $15.46(15)$ \\
Obstetric & $84.54(82)$ \\
\hline
\end{tabular}

Table 2: Distribution of wound dehiscence in elective and emergency surgeries

\begin{tabular}{ll}
\hline Surgery & Percentage $(n=97)$ \\
\hline Elective & $18.56(18)$ \\
Emergency & $81.44(79)$ \\
\hline
\end{tabular}

Table 3A: Distribution of wound dehiscence cases in different age groups (obstetric cases)

\begin{tabular}{lc}
\hline Age & Percentage $(n=82)$ \\
\hline$<20$ & $7.31(6)$ \\
$20-29$ & $82.92(68)$ \\
$30-39$ & $9.75(8)$ \\
$40-49$ & 0 \\
$>50$ & 0 \\
Total & 100.00 \\
\hline
\end{tabular}

Table 3B: Distribution of wound dehiscence cases in different groups (gynecological cases)

\begin{tabular}{lc}
\hline Age & Percentage $(n=15)$ \\
\hline$<20$ & 0 \\
$20-29$ & $13.33(2)$ \\
$30-39$ & $33.33(5)$ \\
$40-49$ & $40.00(6)$ \\
$>50$ & $13.33(2)$ \\
Total & 100.00 \\
\hline
\end{tabular}

\section{Risk Factors for Wound Dehiscence}

Age

Among 82 wound dehiscence cases who underwent obstetric surgeries, $82.92 \%$ women were in age group of $20-29$ years, $9.75 \%$ were in $30-39$ years, and $7.31 \%$ were less than 20 years of age. Among 15 cases who underwent gynecological surgeries, 40\% belonged to age group of $40-49$ years, $33.33 \%$ to $30-39$ years, $13.33 \%$ to more than 50 years, and $13.33 \%$ to less than 20 years of age (Tables $3 \mathrm{~A}$ and $3 \mathrm{~B}$ ).

\section{Parity}

Among obstetric cases, primipara had a higher number of wound dehiscence cases with 53 patients (54.64\%). Twenty-nine patients were multipara (35.36\%). Among gynecological cases, the incidence was lowest in nullipara and grandmultipara (Tables $4 \mathrm{~A}$ and $4 \mathrm{~B}$ ).

\section{Indications for LSCS}

The two commonest indications for LSCS among wound dehiscence patients were fetal distress (25.77\%) and previous LSCS (19.58\%). The least common incidence was twin gestation (Table 5).

Antepartum hemorrhage includes central placenta previa and abruption both. Hypertension includes gestational hypertension, preeclampsia (mild and severe), and chronic hypertension. Previous LSCS includes both previous 1 and previous 2 . 
Incidence of Wound Dehiscence Following Obstetric and Gynecological Surgeries

Table 4A: Paritywise distribution of wound dehiscence cases (obstetric cases)

\begin{tabular}{lc}
\hline Parity & Percentage $(n=82)$ \\
\hline Primipara & $64.63(53)$ \\
Multipara & $35.36(29)$ \\
P2 & $23.17(19)$ \\
P3 & $10.97(9)$ \\
P4 & $1.21(1)$ \\
PP4 & 0.00 \\
Total & 100.00 \\
\hline
\end{tabular}

Table 4B: Paritywise distribution of wound dehiscence cases (gynecological cases)

\begin{tabular}{lc}
\hline Parity & Percentage $(n=15)$ \\
\hline Nullipara & $6.66(n=1)$ \\
Primipara & 0.00 \\
Multipara & \\
P2 & $33.33(n=5)$ \\
P3 & $13.33(n=2)$ \\
P4 & $26.66(n=4)$ \\
PP4 & $20.0(n=3)$ \\
Total & 100.00 \\
\hline
\end{tabular}

Table 5: Indications for LSCS among wound dehiscence cases

\begin{tabular}{lc}
\hline Indication & Percentage $(n=82)$ \\
\hline Fetal distress & $30.48(n=25)$ \\
Previous LSCS & $23.17(n=19)$ \\
Hypertension & $15.85(n=13)$ \\
CPD & $8.53(n=7)$ \\
Malpresentation & $6.09(n=5)$ \\
Failed induction & $3.65(n=3)$ \\
Antepartum hemorrhage & $2.43(n=2)$ \\
Preterm labor & $2.43(n=2)$ \\
MCDA twins & $1.21(n=1)$ \\
Total & 100.00 \\
\hline
\end{tabular}

\section{Comorbidities}

The most common comorbidity was hypertension (19\%) among wound gape patients. Hypertension included gestational hypertension, preeclampsia (mild and severe), HELLP, and chronic hypertension. The other comorbidities were diabetes $(5.15 \%)$ including GDM and PROM (5.15\%) (Table 6).

\section{Anemia}

As a risk factor, anemia was evaluated separately. In our study, all patients were found to have anemia, which varied from mild to moderate. AHb level $<11 \mathrm{~g} / \mathrm{dL}$ was taken as anemia. Majority of the patients had $\mathrm{Hb}$ levels between 10 and $10.9 \mathrm{~g} / \mathrm{dL}$, which could be classified as mild anemia according to ICMR classification.

The total number of mildly anemic patients was 76 (78.35\%). While a total number of 21 patients had moderate anemia, that is, Hb was $79.9 \mathrm{~g} / \mathrm{dL}$ (Table 7).

\section{Previous Surgeries}

Any history of previous surgeries was also taken into account as one of the predisposing factors. About $14 \%$ of patients gave such history (Table 8).
Table 6: Various coexisting risk factors in wound dehiscence cases

\begin{tabular}{lc}
\hline Risks & Percentage $(n=97)$ \\
\hline Nil & $56.70(55)$ \\
Hypertension & $19.58(19)$ \\
GDM/overt DM/IGT & $5.15(5)$ \\
PROM & $5.15(5)$ \\
Fever on admission & $1.03(1)$ \\
Total & 100.00 \\
\hline
\end{tabular}

Table 7: $\mathrm{Hb}$ levels in wound dehiscence cases

\begin{tabular}{lc}
\hline$H b(g \%)$ & Percentage $(n=97)$ \\
\hline $7-9.9$ & $21.65(21)$ \\
$10-10.9$ & $78.35(76)$ \\
Total & 100.00 \\
\hline
\end{tabular}

Table 8: History of previous surgeries

\begin{tabular}{lc}
\hline Previous surgery & Percentage $(n=97)$ \\
\hline None & $76.29(74)$ \\
Previous surgery & $14.43(14)$ \\
Total & 100.00 \\
\hline
\end{tabular}

Table 9A: Hospital stay (for primary surgery, secondary suturing, wound healing)

\begin{tabular}{lc}
\hline Hospital stay (no. of days) & Percentage $(n=60)$ \\
\hline $0-7$ & $0(0)$ \\
$8-14$ & $15(9)$ \\
$15-21$ & $45(27)$ \\
$22-28$ & $36.66(22)$ \\
$29-35$ & $3.3(2)$ \\
Total & 100.00 \\
\hline
\end{tabular}

Table 9B: Hospital stay [for secondary suturing, wound healing (women discharged after primary surgery and readmitted with wound dehiscence)]

\begin{tabular}{lc}
\hline Hospital stay (no. of days) & Percentage $(n=37)$ \\
\hline $0-7$ & $16.21(6)$ \\
$8-14$ & $40.5(15)$ \\
$14-21$ & $43.24(16)$ \\
Total & 100.00 \\
\hline
\end{tabular}

\section{Average Duration of Hospital Stay}

Among 97, 60 patients developed wound dehiscence while their postoperative stay in the hospital following primary surgery and continued hospital stay further to undergo secondary suturing. The hospital stay for these patients even extended over 30 days (Table 9A). However, 37 patients out of 97 were discharged from the hospital with an apparently healthy wound and got readmitted in view of wound dehiscence for secondary suturing. The average hospital stay was shorter for these patients (Table 9B).

\section{Causative Organisms}

All the wound dehiscence cases were evaluated for the presence of infection through swabs taken from the wound sites and then sent for culture and sensitivity in the microbiology laboratory in the hospital. Most of the cultures showed absence of any organism or 
presence of skin commensals. Apart from these the most commonly isolated organism were Staphylococcus aureus and E. coli (Table 10A). There was also a comparison done between commonly isolated organisms in emergency cases and elective cases (Tables 10B and 10C). However, the isolated organisms were similar in both the cases.

Table 10A: Organisms isolated from the wound site

\begin{tabular}{ll}
\hline Wound culture & Percentage $(n=97)$ \\
\hline No organisms & $23.71(23)$ \\
Skin commensals & $22.68(22)$ \\
S. aureus & $13.40(13.4)$ \\
E. coli & $10.31(10)$ \\
Pseudomonas aeroginosa & $8.25(8)$ \\
Betalactamase-producing citrobacter & $2.06(2)$ \\
Betalactamase-producing E. coli & $1.03(1)$ \\
Citrobacter & $2.06(2)$ \\
Citrobacter, E. coli & $1.03(1)$ \\
Coagulase neg S. aureus & $1.03(1)$ \\
Enterococcus species & $1.03(1)$ \\
ESBL-producing citrobacter & $2.06(2)$ \\
Klebsiella pneumoniae & $4.12(4)$ \\
Klebsiella pneumoniae, Pseudomonas & $1.03(1)$ \\
MRSA & $5.15(5)$ \\
S. aureus, E. coli & $1.03(1)$ \\
\hline
\end{tabular}

Table 10B: Organisms isolated from the wound site (elective cases)

\begin{tabular}{lc}
\hline Wound culture & Percentage $(n=18)$ \\
\hline No organisms & $41.11(7)$ \\
Skin commensals & $27.77(5)$ \\
S. aureus & $5.5(1)$ \\
E. coli & $5.5(1)$ \\
Betalactamase-producing E. coli & $5.5(1)$ \\
Klebsiella pneumoniae & $11.11(2)$ \\
MRSA & $5.5(1)$ \\
\hline
\end{tabular}

Table 10C: Organisms isolated from the wound site (emergency cases)

\begin{tabular}{lc}
\hline Organisms isolated from wound site & Percentage $(n=74)$ \\
\hline No organisms & $21.62(16)$ \\
Skin commensals & $22.97(17)$ \\
S. aureus & $16.21(12)$ \\
E. coli & $8.1(6)$ \\
Pseudomonas aeuroginosa & $10.8(8)$ \\
MRSA & $5.4(4)$ \\
Betalactamase-producing citrobacter & $2.7(2)$ \\
Citrobacter & $2.7(2)$ \\
ESBL-producing citrobacter & $1.35(1)$ \\
Citrobacter, E. coli & $1.35(1)$ \\
Coagulase neg S. aureus & $1.35(1)$ \\
Enterococcus species & $1.35(1)$ \\
Klebsiella pneumoniae & $1.35(1)$ \\
Klebsiella pneumoniae, pseudomonas & $1.35(1)$ \\
S. aureus, E. coli & $1.35(1)$ \\
\hline
\end{tabular}

\section{Discussion}

Postoperative wound infection and dehiscence is an upcoming challenge to the operating surgeon. The success in the field of surgery in terms of advances in surgical techniques and the easy availability of surgery as a treatment option have been marred by the increasing incidence of wound dehiscence. Wound healing by itself is a complex process affected by various internal and external factors. The internal factors constitute comorbidities like diabetes and hypertension. The external factors include wound infection, the common suturing practices, suture materials, and wound care. The incidence of infection in post-cesarean wound and independent risk factors related with infection of wound have been studied retrospectively at tertiary care hospitals. ${ }^{7}$ The overall incidence of wound dehiscence in our hospital setting was found to be $3.05 \%$ and all of these patients required secondary suturing. An introspection of these wound dehiscence cases led to the initiation of this retrospective study. The preoperative preparation, the wound care postoperatively, suture materials used, and the suturing technique or practice differ from one institute to another. The practice of changing dressing postoperatively too is different in different institutes. The normal practice is to change the dressing on the 2 nd and 3 rd postoperative day as a routine in our institute. The change of dressing was done in postnatal wards.

Intraoperatively, the subcutaneous fat suturing was not done and directly subcuticular sutures were taken in majority of the cases in our institute. Abdomen closure in our institute was done with polyglactin. The rectus sheath was closed by polyglactin no. 1 and skin by polyglactin 2-0,3-0. Since wound healing is affected by various factors like comorbidities, presence of infection, and wound care, the secondary outcomes in this study were all those risk factors that could play a role in causing wound dehiscence. In our study, the commonest coexisting morbidities were found to be hypertension and diabetes. Among LSCS cases, patients who developed wound dehiscence had commonest indication for section as fetal distress followed by hypertension. Among coexisting diseases, hypertension and diabetes showed to have a greater incidence of wound dehiscence. Another secondary outcome was the average stay of patients with wound dehiscence, which reflected overall morbidity in such patients. Patients who developed wound dehiscence during their stay in the hospital had a longer stay in the hospital. It was important to note the coexistence of any infection in the wounds. However, most of the wound dehiscence culture came negative for presence of any organisms. The second most common wound culture was skin commensals. However, S. aureus and E. coli were the most frequently isolated organism from the infected wounds. Reasons that enhance risk to postoperative wound dehiscence incorporate age, obesity, nutritional status, preoperative medical disorders like anemia, diabetes, jaundice, use of steroids, poor intraoperative preparation, emergency or elective surgeries, duration and type of surgeries, postoperative wound site contamination or increase in intra-abdominal pressure, infection, etc. ${ }^{4}$

Skilled appraisal and a methodical consideration of the physiological needs for wound healing allow to appreciate why some wounds heal quickly and others are slow to heal. ${ }^{9}$ Earlier researchers have proposed that antimicrobial prophylaxis reduces the peril of endometritis and incisional SSI when correctly administered. ${ }^{10}$

Clinicians have observed that incidence of postoperative wound gaping was higher in emergency obstetrics cases in contrast 
to elective cases. ${ }^{11}$ Work has been carried out to investigate causal features regarding abdominal wound dehiscence and evisceration in order to ameliorate healing. ${ }^{12}$

Incidence of wound dehiscence following obstetric and gynecological surgeries can be taken as surgical emergency, which can extend the total period of sickness causing unnecessary stress to patients and can also accelerate the hospital expenditures. There is a strong need to avoid these impediments by properly addressing the factors incorporated in the development of wound dehiscence. ${ }^{4}$ The wound gaping problem has started occupying scientific concern revealed by different explorations conducted from time to time. Clinicians have used various combinations of antibiotics as a single-dose chemoprophylaxis in gynecological surgery. ${ }^{13}$ Concerning the fact that vitamin $C$ plays an important role in maintaining the integrity of capillaries, investigations have been carried out relating vitamin $C$ deficiency and wound healing. ${ }^{14}$ Wound closure has a great influence on wound healing in gynecological surgery. ${ }^{15}$ Though wound dehiscence is a surgical snag, however, factors associated with it are knowable and preventable. Triumph of a gynecological practice rests on wary incision and appropriate closure of the wound. Various factors are to be taken care of to check postoperative infections and wound dehiscence.

Scientists believe that apposite surgical wear and proper hand washing alongwith adequate theater sterility are considered to be significant aspects in minimizing the infections. In order to avoid wound dehiscence, accurate surgical procedures involving maintenance of effective homeostasis, removal of all devitalized tissue along with tender tissue dealing and exterminating dead space, circumventing unintentional entries into a viscous, employing drains and suture material aptly are important. ${ }^{16}$ Wound dehiscence has many causes and deterrence is of prime importance for bringing reduction in incidence of wound dehiscence. Scientists consider that patients who develop wound dehiscence have more chances to spend time in an ICU. ${ }^{17}$ Perceptiveness of the different treatment choices and timely referral guides to Esthetic and good functional outcomes. ${ }^{18}$ It is important to correlate wound gaping with certain disease states like diabetes. ${ }^{19}$ Earlier research attempted to compare healing in postoperative wounds with occlusive gauze dressing. $^{20}$

The present investigation has attempted to provide incidence of wound dehiscence following obstetric and gynecologic surgeries at a tertiary hospital and advocated the avoidance of changing of dressing in the wards prior to the 5th postoperative day. This will assist in discerning the relevance of appraisals incorporating suturing subcutaneous fat before approximating the skin with maintaining drain in situ in obese women. The strength of this study includes the number of participating patients, the appraised risk factors, and the most of the wound gape culture came negative for presence of any organisms.

\section{Conclusion}

Wound healing is pretentious not just by patient issues but also by intraoperative and postoperative factors. Apart from following the standard operative modus operandi, early wound dressing (postoperative day 3 ) is a vital aspect piloting to enhanced wound gape. To lessen this occurrence, dressing should almost certainly be carried out first only on the 5th postoperative day, which may avert establishment of infection in the wound from wards or may thwart commotion of healing process, imparting a better healed wound on the 5th postoperative day. Moreover, subcutaneous fat should possibly be sutured intraoperatively, which may put off creation of dead space for secretions to get stockpiled that may prompt the wound dehiscence or cause wound infection. Besides, patient elements incorporating hypertension and diabetes could be supervised efficiently to put a stop to wound dehiscence. Convention of pre- and postoperative antibiotics, timing of preoperative antibiotics, reducing second stage of labor, proper handling of sterile instruments, intra-op suturing techniques and materials, postoperatively proper wound care practices by doctors and the allied staff, and usage of clean linen in wards are crucial in bringing down the overall number of wound dehiscence.

\section{Clinical Significance}

Intraoperative and postoperative factors are noteworthy in wound healing in addition to the patient factor. Routine dressing should probably be done first only on the 5th postoperative day, which may prevent introduction of infection in the wound from wards or may prevent disruption of the healing process. However, dressing can be done earlier than 5th postoperative day when there is soakage of dressing or there are signs of sepsis. Subcutaneous fat should probably be sutured intraoperatively. Early and effective management of patient factors can assist in preventing wound dehiscence and can prove worthwhile for improved result of the surgery alongwith curtailing off the overall burden of wound dehiscence. The data presented in the investigation can support in ameliorating the understanding of the patient-associated factors in wound dehiscence cases. This can provide assistance to surgical teams in directing the patients of their risk before starting the surgery. Surgeons can also focus on modifiable risk factors before going to elective surgeries. Clinically, wound dehiscence following obstetric and gynecological surgeries is posing a challenge; yet, it is manageable employing scientific approaches.

\section{References}

1. Clark JJ. Wound repair and factors influencing healing. Critic Care Nurs Q 2002;25(1):1-2. DOI: 10.1097/00002727-20020500000002.

2. Colp R. Disruption of abdominal wounds. Annal Surg 1934;99(1):14-27. DOI: 10.1097/00000658-193401000-00003.

3. Gantwerker EA, Hom DB. Skin: histology and physiology of wound healing. Clin Plast Surg 2011;19(3):441-453. DOI: 10.1016/ j.fsc.2011.06.009.

4. Narang R, Nandmer GK, Sapkal R. Factors affecting post operative wound gaping and their outcome in obstetrical and gynecological abdominal surgeries. Int J Reprod Contracept Obstet Gynecol 2017;6(4):1530-1533.

5. Bharatnur S, Agarwal V. Assessment of risk factors for surgical site infection in obstetric patients and its impact on postoperative recovery. Indian J Obstet Gynecol 2018;6:59-64. DOI: 10.21088/ ijog.2321.1636.6118.11.

6. Pathak A, Mahadik K, Swami MB, et al. Incidence and risk factors for surgical site infections in obstetric and gynecological surgeries from a teaching hospital in rural India. Antimicrob Resist Infect Control 2017;6:66. DOI: 10.1186/s13756-017-0223-y.

7. Al Jama FE. Risk factors for wound infection after lower segment caesarean section. Qatar Med J 2012;12(2):26-31.

8. Arti PJ, Shuchi VP. Study of wound infection: major morbidity after cesarean section. Global J Res Analysis Intern 2014;3(7).

9. Khandra HP, Vyas PH, Patel NJ, et al. Factors affecting postoperative laparotomy wound complications. Int Archiv Integra Med 2015;2(1):71-75. 
10. Baaqeel $H$, Baaqeel R. Timing of administration of prophylactic antibiotics for caesarean section: a systematic review and metaanalysis. BJOG 2013;120(6):661-669. DOI: 10.1111/1471-0528. 12036.

11. Malhotra R, Walia GA. Study on factors affecting post operative wound infection. Int J Com Health and Med Res 2015;1(1): 17-21.

12. Haddad V, Macon W. Abdominal wound dehiscence and evisceration: contributing factors and improved mortality. Am Surgeon 1980;46(9):508-513.

13. Houang ET, Watson C, Howell R, et al. Ampicillin combined with sulbactam or metronidazole for single-dose chemoprophylaxis in major gynaecological surgery. J Antimicro Chemoth 1984;14(5): 529-535. DOI: 10.1093/jac/14.5.529.

14. Lanman TH, Ingalls TH. Vitamin C deficiency and wound healing: an experimental and clinical study. Annals Surg 1937;105(4):616. DOI: 10.1097/00000658-193704000-00015.
15. Boesch CE, Umek W. Effects of wound closure on wound healing in gynecologic surgery: a systematic literature review. J Reproduc Med 2009;54(3):139-144.

16. Mahana S, Biswas S. An analytical study on wound dehiscence and related factors. Int J Reprod Contracept Obstet Gynecol 2013;2(4):506-508. DOI: 10.5455/2320-1770.ijrcog20131203.

17. Liau KH, Aung KT, Chua N, et al. Outcome of a strategy to reduce surgical site infection in a tertiary-care hospital. Surg Infect (Larchmt) 2010;11(2):151-159. DOI: 10.1089/sur.2008.081.

18. Patil SB, Harsh S, Nikam R, et al. Post caesarean section wound gaping: Discussing the problem and treatment options. J Med Dental Sci Res 2016;11(3):43-46.

19. Alberti KG, Thomas DJ. The management of diabetes during surgery. Br J Anaesth 1979;51(7):693-710. DOI: 10.1093/bja/51.7.693.

20. Sharma AS, Yadav D, Bindal J. A study to compare healing in postoperative wounds with occlusive gauze dressing and after Omitting the dressing. JMSCR 2015;3:5734-5741. 